Mathematical Research Letters 1, 729-737 (1994)

\title{
ON STRICHARTZ AND EIGENFUNCTION ESTIMATES FOR LOW REGULARITY METRICS
}

\author{
Hart F. Smith and Christopher D. Sogge
}

\begin{abstract}
A в s т R A т. We produce, for dimensions $n \geq 3$, examples of wave operators for which the Strichartz estimates fail. The examples include both Lipschitz and $C^{1, \alpha}$ metrics, for each $0<\alpha<1$, where by the latter we mean that the gradient satisfies a Hölder condition of order $\alpha$. We thus conclude that, on the scale of Hölder regularity, an assumption of at least 2 bounded derivatives for the metric (i.e., $C^{1,1}$ ) is necessary in order to assure that the Strichartz estimates hold. The same construction also yields, for dimensions $n \geq 2$, second order elliptic operators with Lipschitz or $C^{1, \alpha}$ coefficients for which certain eigenfunction estimates, established by the second author for operators with $C^{\infty}$ coefficients, fail to hold.
\end{abstract}

\section{Strichartz Estimates}

We recall here the global version of the Strichartz estimates on $\mathbb{R}^{1+n}$, for the Euclidean wave operator $\square=\partial_{t}^{2}-\sum_{j=1}^{n} \partial_{j}^{2}$. These state (see $[\operatorname{St1}, 2]$ ) that, if $u$ solves the system of equations

$$
\left\{\begin{array}{l}
\square u=F \in L^{\frac{2(n+1)}{n+3}}\left(\mathbb{R}^{1+n}\right), \\
u(0, \cdot)=f \in H^{\frac{1}{2}}\left(\mathbb{R}^{n}\right), \\
\partial_{t} u(0, \cdot)=g \in H^{-\frac{1}{2}}\left(\mathbb{R}^{n}\right),
\end{array}\right.
$$

then the following estimate holds

$$
\|u\|_{L^{\frac{2(n+1)}{n-1}}\left(\mathbb{R}^{1+n}\right)} \leq C_{n}\left(\|f\|_{H^{\frac{1}{2}\left(\mathbb{R}^{n}\right)}}+\|g\|_{H^{-\frac{1}{2}\left(\mathbb{R}^{n}\right)}}+\|F\|_{L^{\frac{2(n+1)}{n+3}\left(\mathbb{R}^{1+n}\right)}}\right) .
$$

Here, $H^{s}\left(\mathbb{R}^{n}\right)$ denotes the homogeneous Sobolev space of functions with $s$ fractional derivatives in $L^{2}\left(\mathbb{R}^{n}\right)$. For variable coefficient wave equations with sufficiently regular coefficients, this estimate holds locally (that is,

The authors were supported in part by the National Science Foundation.

Received August 14, 1994. 
over compact regions of space and time that are consistent with causality); see $[\mathrm{K}]$ and $[\mathrm{MSS}]$. We show that even the local estimate can fail for low regularity metrics if $n \geq 3$.

The counterexample consists of producing a traveling wave packet that is more highly concentrated than is possible for smooth metrics. Roughly speaking, the singularities of the metric produce a sharper focusing of the packet. To compare our example to the free case, we remark that the Strichartz estimate (1.1) is seen to be sharp by letting $u_{\lambda}(t, x, y)=$ $\cos (t \sqrt{-\Delta}) \varphi_{\lambda}(x, y)$, where $x \in \mathbb{R}, y \in \mathbb{R}^{n-1}$ and

$$
\varphi_{\lambda}(x)=\varphi\left(\lambda x, \lambda^{\frac{1}{2}} y\right)
$$

with $\varphi$ being a Schwartz function with Fourier transform supported in a small ball about the point $(1,0, \ldots, 0)$. The function $u_{\lambda}(t, x, y)$ is, for small t, essentially a bump function concentrated in the set

$$
|x-t| \leq \lambda^{-1}, \quad|y| \leq \lambda^{-\frac{1}{2}}
$$

The two sides of (1.1) are then comparable for all $\lambda$, and letting $\lambda$ tend to $\infty$ shows that the estimates are sharp.

For examples of Lipschitz and $C^{1, \alpha}$ metrics, we produce a wave packet $u_{\lambda}(t, x, y)$, constructed of frequencies of size $\lambda$, concentrated in the region

$$
|x-t| \leq \lambda^{2 \delta-2}, \quad|y| \leq \lambda^{-\delta}
$$

where $\delta>\frac{1}{2}$. If $n \geq 4$, then the volume of this region is strictly less than in the free case. In case $n=3$, we use the sharper fact that as $t \rightarrow 0$ the support of our packet becomes smaller, to a degree which still contradicts the Strichartz estimates, although the failure is only logarithmic in $\lambda$. Notice that for $n=2$ the volume of this region is greater than in the free case, and this construction therefore does not contradict the Strichartz estimates.

For the example, consider the wave equation

$$
\frac{1}{1-|y|^{1+\alpha}}\left(\Delta_{y}+\partial_{x}^{2}\right) u(t, x, y)=\partial_{t}^{2} u(t, x, y), \quad|y|<1 .
$$

The metric is then $C^{1, \alpha}$ if $0<\alpha<1$, and is Lipschitz if $\alpha=0$. We look for solutions to (1.2) of the form

$$
u(t, x, y)=e^{i x \xi-i t \tau} f(y)
$$


which leads to the differential equation

$$
\Delta_{y} f(y)=\left(\tau^{2}|y|^{1+\alpha}-\left(\tau^{2}-\xi^{2}\right)\right) f(y) .
$$

Let $A_{\alpha}(y)$ denote the normalized ground state for the Schrödinger equation with potential $|y|^{1+\alpha}$

$$
-\Delta_{y} A_{\alpha}(y)+|y|^{1+\alpha} A_{\alpha}(y)=c_{\alpha} A_{\alpha}(y) .
$$

By [RS], Theorems XIII.47, and [A], Theorems 4.1 and 5.1, the function $A_{\alpha}(y)$ is radial, strictly positive, and satisfies

$$
\left|A_{\alpha}(y)\right| \leq C_{N, \alpha} e^{-N|y|}
$$

for all $N>0$. By elliptic regularity, $A_{\alpha}(y) \in C^{3, \alpha}\left(\mathbb{R}^{n-1}\right)$, provided that $0<\alpha<1$. In case $\alpha=0$, then $A_{0}(y) \in C^{2,1-\varepsilon}\left(\mathbb{R}^{n-1}\right)$ for all $\varepsilon>0$.

Written as a function of the radial variable $r$, the following ordinary differential equation is satisfied

$$
\left(\left(r \partial_{r}\right)^{2}+(n-2) r \partial_{r}-r^{3+\alpha}+c_{\alpha} r^{2}\right) A_{\alpha}(r)=0 .
$$

An induction argument yields that

$$
\left|\left(r \partial_{r}\right)^{k} A_{\alpha}(r)\right| \leq C_{k} e^{-r}
$$

for all $k$, and $r>0$.

Now suppose that

$$
\tau^{2}-\xi^{2}=c_{\alpha} \tau^{2 \delta}, \quad \delta=\frac{2}{3+\alpha} .
$$

It follows that

$$
f(y)=A_{\alpha}\left(\tau^{\delta} y\right)
$$

is a solution to (1.3). Equation (1.5) determines $\tau$ as a function of $\xi$ :

$$
\tau=\rho(\xi)=\xi+\frac{c_{\alpha}}{2} \xi^{2 \delta-1}+r(\xi),
$$

where

$$
\left|\partial_{\xi}^{j} r(\xi)\right| \leq C_{j} \xi^{4 \delta-3-j}, \quad \forall j .
$$

For $\lambda$ large and positive, we obtain a solution $u_{\lambda}(t, x, y)$ to $(1.2)$ by superposition:

$$
u_{\lambda}(t, x, y)=\lambda^{(n \delta-\delta-1) / 2} \int e^{-i t \rho(\xi)+i x \xi} \beta\left(\lambda^{-1} \xi\right) A_{\alpha}\left(\rho(\xi)^{\delta} y\right) d \xi,
$$

where $\beta$ is a positive bump function that vanishes outside a small interval about 1 . 
Theorem 1. The function $u_{\lambda}(t, x, y)$ satisfies

$$
\left\{\begin{array}{l}
\left(\left(1-|y|^{1+\alpha}\right) \partial_{t}^{2}-\left(\Delta_{y}+\partial_{x}^{2}\right)\right) u_{\lambda}(t, x, y)=0, \\
\left\|u_{\lambda}(0, x, y)\right\|_{H^{\frac{1}{2}}\left(\mathbb{R}^{n}\right)}+\left\|\partial_{t} u_{\lambda}(0, x, y)\right\|_{H^{-\frac{1}{2}}\left(\mathbb{R}^{n}\right)} \leq C \lambda^{\frac{1}{2}}
\end{array}\right.
$$

where $C$ depends only on $n$ and $\alpha$. On the other hand, if $n=3$,

$$
\int_{0}^{\frac{1}{4}}\left(\int_{|(x, y)| \leq \frac{1}{2}}\left|u_{\lambda}(t, x, y)\right|^{4} d x d y\right) d t \geq C^{-1} \lambda^{2} \ln \left(\lambda^{2 \delta-1}\right),
$$

and if $n \geq 4$,

$$
\int_{0}^{\frac{1}{4}}\left(\int_{|(x, y)| \leq \frac{1}{2}}\left|u_{\lambda}(t, x, y)\right|^{\frac{2(n+1)}{n-1}} d x d y\right) d t \geq C^{-1} \lambda^{\frac{4+2 \delta(n-3)}{n-1}} .
$$

The Strichartz estimates are thus seen to fail for the solution $u_{\lambda}$, since $\delta>\frac{1}{2}$ for $\alpha<1$. We remark that for $n=3$ the failure is only logarithmic in the frequency $\lambda$, whereas for $n \geq 4$ there is a strict power difference. We also remark that one can take either the homogeneous or nonhomogeneous Sobolev norms in (1.7).

Proof of Theorem 1. We begin by establishing (1.7). That $u_{\lambda}$ is a solution of the wave equation follows from the above construction. We next observe that, by Plancherel's Theorem,

$$
\int\left|u_{\lambda}(t, x, y)\right|^{2} d x d y \approx 1
$$

uniformly over $t$ and $\lambda$. By equation (1.2) and the decay estimates on $A_{\alpha}(y)$, we similarly obtain

$$
\int\left|\left(\partial_{x}^{2}+\Delta_{y}\right) u_{\lambda}(t, x, y)\right|^{2} d x d y \approx \lambda^{4}
$$

Interpolation yields that $\left\|u_{\lambda}(0, x, y)\right\|_{H^{\frac{1}{2}\left(\mathbb{R}^{n}\right)}} \lesssim \lambda^{\frac{1}{2}}$. To estimate the $-\frac{1}{2}$ Sobolev norm of $\partial_{t} u_{\lambda}$, we write

$$
\partial_{t} u_{\lambda}(t, x, y)=\partial_{x} v_{\lambda}(t, x, y),
$$

where $\left\|v_{\lambda}(0, x, y)\right\|_{H^{\frac{1}{2}}\left(\mathbb{R}^{n}\right)} \lesssim \lambda^{\frac{1}{2}}$. 
To establish the remaining part of the theorem, we will show that, for $-\frac{1}{4} \leq t \leq \frac{1}{4}$

$$
\int_{|(x, y)| \leq \frac{1}{2}}\left(1+\lambda^{\delta}|y|+\frac{\lambda|x-t|}{1+\lambda^{2 \delta-1}|t|}\right)^{-2}\left|u_{\lambda}(t, x, y)\right|^{2} d x d y \geq C^{-1}
$$

where the constant is independent of $\lambda$ and $t$. By Hölder's inequality, we conclude from (1.9) that

$$
C^{-1} \leq\left[\lambda^{-\delta(n-1)-1}\left(1+\lambda^{2 \delta-1}|t|\right)\right]^{\frac{2}{n-1}} \int_{|(x, y)| \leq \frac{1}{2}}|u(t, x, y)|^{\frac{2(n+1)}{n-1}} d x d y
$$

which immediately yields the desired estimates, after integrating in $t$.

We establish (1.9) by showing that

$$
\int_{\mathbb{R}^{n}}\left(1+\lambda^{\delta}|y|+\frac{\lambda|x-t|}{1+\lambda^{2 \delta-1}|t|}\right)^{2}\left|u_{\lambda}(t, x, y)\right|^{2} d x d y \leq C
$$

An application of the Schwarz inequality, together with (1.8), shows that this implies (1.9) for large $\lambda$. Notice that this inequality quantifies the concentration mentioned before.

To establish (1.10), we write

$$
\begin{aligned}
& u_{\lambda}(t, x, y) \\
& \quad=\lambda^{(n \delta-\delta-1) / 2} \int e^{i(x-t) \xi-i \frac{1}{2} c_{\alpha} t \xi^{2 \delta-1}} e^{-i t r(\xi)} \beta\left(\lambda^{-1} \xi\right) A_{\alpha}\left(\rho(\xi)^{\delta} y\right) d \xi .
\end{aligned}
$$

We make use of the following estimates:

$$
\left|\partial_{\xi}^{j}\left(e^{-i \operatorname{tr}(\xi)} \beta\left(\lambda^{-1} \xi\right) A\left(\rho(\xi)^{\delta} y\right)\right)\right| \leq \lambda^{-j} e^{-\lambda^{\delta}|y|},
$$

which follow by (1.4) and (1.6).

If $|t| \leq \lambda^{1-2 \delta}$, then in addition we have

$$
\left|\partial_{\xi}^{j} e^{-i \frac{1}{2} c_{\alpha} t \xi^{2 \delta-1}}\right| \leq C_{j} \lambda^{-j}
$$

on the support of $\beta\left(\lambda^{-1} \xi\right)$, and it follows easily that

$$
\left|u_{\lambda}(t, x, y)\right| \leq C_{N} \lambda^{(n \delta-\delta+1) / 2}\left(1+\lambda^{\delta}|y|+\lambda|x-t|\right)^{-N},
$$


which implies (1.10) in this case. For $|t| \geq \lambda^{1-2 \delta},(1.10)$ is a consequence of the following estimate

$$
\left|u_{\lambda}(t, x, y)\right| \leq C_{N} \lambda^{(n \delta-\delta+1) / 2}\left(\lambda^{2 \delta-1}|t|\right)^{-1 / 2}\left(1+\lambda^{\delta}|y|+\frac{\lambda|x-t|}{\lambda^{2 \delta-1}|t|}\right)^{-N} .
$$

To establish (1.13), we write

$$
\begin{array}{r}
u_{\lambda}(t, x, y)=\lambda^{(n \delta-\delta+1) / 2} \int e^{i \lambda(x-t) \sigma-i \frac{1}{2} c_{\alpha} t \lambda^{2 \delta-1} \sigma^{2 \delta-1}} e^{-i \operatorname{tr}(\lambda \sigma)} \\
\beta(\sigma) A_{\alpha}\left(\rho(\lambda \sigma)^{\delta} y\right) d \sigma .
\end{array}
$$

Suppose that $\lambda|x-t| \leq C \lambda^{2 \delta-1}|t|$. Then the phase takes the form

$$
\left(t \lambda^{2 \delta-1}\right)\left[\frac{c_{\alpha}}{2} \sigma^{2 \delta-1}+\varepsilon \sigma\right]
$$

where $|\varepsilon| \leq C$. The term in brackets has nonvanishing second derivative on the support of $\beta(\sigma)$, so that (1.13) follows by stationary phase and the estimates (1.11). On the other hand, if $\lambda|x-t| \geq C \lambda^{2 \delta-1}|t|$, then we write the phase as

$$
\lambda(x-t)\left[\sigma+\varepsilon \frac{c_{\alpha}}{2} \sigma^{2 \delta-1}\right],
$$

where $|\varepsilon| \leq C^{-1}$. For $C$ sufficiently large, the term in brackets has nonvanishing derivative on the support of $\beta(\sigma)$. Integration by parts shows that the estimates (1.12) hold in this case, which implies (1.13) since $\lambda|x-t| \geq C \lambda^{2 \delta-1}|t| \geq 1$.

\section{Eigenfunction Estimates}

Suppose that $P$ is a positive, second order elliptic differential operator on a compact $n$-dimensional manifold $M^{n}$, which is self-adjoint with respect to some smooth density, and suppose that $\left\{e_{\mu}\right\}$ is an orthonormal basis for $L^{2}\left(M^{n}\right)$ consisting of eigenfunctions for $P$ :

$$
P e_{\mu}=\mu^{2} e_{\mu} .
$$

For $\lambda \in \mathbb{R}$, let $\chi_{\lambda} f$ denote the orthogonal projection of a function $f$ onto the space of eigenfunctions with frequencies in the range $[\lambda, \lambda+1)$ :

$$
\chi_{\lambda} f=\sum_{\mu \in[\lambda, \lambda+1)}\left(e_{\mu}, f\right) e_{\mu} .
$$


It was shown by the second author in [S] that, if the coefficients of $P$ belong to $C^{\infty}\left(M^{n}\right)$, then the following estimates hold.

$$
\left\|\chi_{\lambda} f\right\|_{L^{q}\left(M^{n}\right)} \leq C \lambda^{n\left(\frac{1}{2}-\frac{1}{q}\right)-\frac{1}{2}}\|f\|_{L^{2}\left(M^{n}\right)}, \quad \frac{2(n+1)}{n-1} \leq q \leq \infty .
$$

In [D], Davies showed that these estimates can fail for $q=\infty$ if the coefficients of $P$ are bounded but not continuous. It is then natural to seek counterexamples for smoother coefficients. In his thesis [G], Grieser observed that, for eigenfunctions on the unit disc in $\mathbb{R}^{2}$ with Dirichlet conditions, the estimates (2.1) fail if $q<8$. (In [S] it is assumed that the manifold $M^{n}$ does not have a boundary.) By attaching two copies of the disc along their boundaries, one obtains a compact manifold without boundary, where the coefficients of $P$ are Lipschitz, such that (2.1) fails for $q<8$.

We extend these results by producing, for each $0 \leq \alpha<1$ and each $n \geq 2$, an operator $P$ on the $n$-dimensional torus $\mathbb{T}^{n}$, whose coefficients belong to $C^{1, \alpha}\left(\mathbb{T}^{n}\right)$ if $0<\alpha<1$ and Lip $\left(\mathbb{T}^{n}\right)$ if $\alpha=0$, and such that the estimates (2.1) fail if

$$
q<2+\frac{2}{(n-1)(1-\delta)}, \quad \delta=\frac{2}{3+\alpha} .
$$

To describe the example, let $(x, y)$ denote variables in $(-\pi, \pi] \times(-\pi, \pi]^{n-1}$, which we take as coordinates on $\mathbb{T} \times \mathbb{T}^{n-1}$. For the standard metric on $\mathbb{T}^{n}$, the estimates (2.1) are seen to be sharp by constructing, from frequencies in the range $[\lambda, \lambda+1)$, a function concentrated in the tubular region $|y| \leq \lambda^{-\frac{1}{2}}$. For metrics similar to those of the previous section, we construct approximate eigenfunctions concentrated in the tubular region $|y| \leq \lambda^{-\delta}$. This yields counterexamples to (2.1) for all $n \geq 2$, as opposed to the previous section where the spreading of the wave packet in the $x$ direction, caused by superimposing a range of frequencies, produced counterexamples to the Strichartz estimates only for $n \geq 3$.

Let $c(y)$ be a strictly positive function on $\mathbb{T}^{n-1}$ such that

$$
c^{2}(y)=\frac{1}{1-|y|^{1+\alpha}}, \quad|y| \leq \frac{3}{4},
$$

and such that $c(y)$ belongs to $C^{\infty}\left(\mathbb{T}^{n-1}\right)$ away from the point $y=0$. The operator

$$
P\left(y, \partial_{x}, \partial_{y}\right)=-c(y)^{2}\left(\Delta_{y}+\partial_{x}^{2}\right),
$$

with domain $H^{2}\left(\mathbb{T}^{n}\right)$, is then self adjoint on $L^{2}\left(\mathbb{T}^{n}\right)$ with respect to the measure $c(y)^{-2} d x d y$. The resolvent of $P$ is easily seen to be compact, so that $L^{2}\left(\mathbb{T}^{n}\right)$ admits an orthonormal basis of eigenfunctions for $P$. 
Fix $\psi(y) \in C^{\infty}\left(\mathbb{T}^{n-1}\right)$, such that

$$
\psi(y)= \begin{cases}1, & |y| \leq \frac{1}{2} \\ 0, & |y| \geq \frac{3}{4}\end{cases}
$$

and for positive integers $k$ consider the function

$$
f_{k}(x, y)=k^{(n-1) \delta / 2} e^{i k x} \psi(y) A_{\alpha}\left(\rho(k)^{\delta} y\right) .
$$

We take $k$ large so that $k \leq \rho(k) \leq k+\frac{1}{4}$. It is easily verified that

$$
\left\|f_{k}\right\|_{L^{q}\left(\mathbb{T}^{n}\right)} \approx k^{(n-1) \delta\left(\frac{1}{2}-\frac{1}{q}\right)} .
$$

By the constructions of the previous section, we have

$$
-c^{2}(y)\left(\Delta_{y}+\partial_{x}^{2}\right) f_{k}(x, y)=\rho(k)^{2} f_{k}(x, y)+r_{k}(x, y),
$$

where the error term $r_{k}(x, y)$ vanishes unless $\frac{1}{2} \leq|y| \leq \frac{3}{4}$, and where for all multi-indices $\gamma$ the following holds

$$
\left|\partial_{x, y}^{\gamma} r_{k}(x, y)\right| \leq C_{\gamma} e^{-\frac{1}{2} k^{\delta}}
$$

If it were the case that $r_{k} \equiv 0$, then by taking $\lambda=k$ it would follow immediately from (2.3) that the estimates (2.1) fail if $q$ is as in (2.2). That this failure occurs even though $r_{k}$ need not vanish identically is a result of the following theorem.

Theorem 2. Let $f_{k}(x, y)$ be as above. Then, if $|\lambda-k| \geq 2$, it follows that

$$
\left\|\chi_{\lambda} f_{k}\right\|_{L^{2}\left(\mathbb{T}^{n}\right)} \leq C_{N}\left(1+\lambda^{2}\right)^{-N} e^{-\frac{1}{2} k^{\delta}}, \quad \forall N
$$

As a consequence, the estimate

$$
\left\|\chi_{\lambda} f_{k}\right\|_{L^{q}\left(\mathbb{T}^{n}\right)} \leq C \lambda^{n\left(\frac{1}{2}-\frac{1}{q}\right)-\frac{1}{2}}\left\|\chi_{\lambda} f_{k}\right\|_{L^{2}\left(\mathbb{T}^{n}\right)}
$$

cannot hold with $C$ independent of $\lambda$ and $k$ if

$$
q<2+\frac{2}{(n-1)(1-\delta)}, \quad \delta=\frac{2}{3+\alpha} .
$$

Proof. Let us first show that (2.5) implies the desired consequence. It follows from (2.3) and Minkowski's inequality that

$$
\sum_{j=1}^{\infty}\left\|\chi_{j} f_{k}\right\|_{L^{q}\left(\mathbb{T}^{n}\right)} \geq c k^{(n-1) \delta\left(\frac{1}{2}-\frac{1}{q}\right)} .
$$


If (2.6) holds for $q$, then by (2.5) the left hand side is dominated by $k^{n\left(\frac{1}{2}-\frac{1}{q}\right)-\frac{1}{2}}$, which yields a contradiction in case $q$ is as in $(2.2)$.

To establish (2.5), suppose that $e_{\mu}$ is an eigenfunction with eigenvalue $\mu^{2}$, with $\mu \in[\lambda, \lambda+1)$ and $|\lambda-k|>2$. We then have

$$
\begin{aligned}
& \int f_{k}(x, y) e_{\mu}(x, y) c(y)^{-2} d x d y \\
& \quad=\frac{1}{\mu^{2}-\rho(k)^{2}} \int r_{k}(x, y) e_{\mu}(x, y) c(y)^{-2} d x d y \\
& \quad=\frac{\left(1+\mu^{2}\right)^{-N}}{\mu^{2}-\rho(k)^{2}} \int\left(1-c(y)^{2}\left(\Delta_{y}+\partial_{x}^{2}\right)\right)^{N} r_{k}(x, y) e_{\mu}(x, y) c(y)^{-2} d x d y
\end{aligned}
$$

where the integration by parts is valid since $r_{k}(x, y)$ is supported in the region where $c(y)$ is smooth. Notice that $\mu^{2}-\rho(k)^{2}$ is bounded from below. Therefore, orthogonality and (2.4) yield

$$
\begin{aligned}
\left\|\chi_{\lambda} f_{k}\right\|_{L^{2}} & \leq C\left(1+\lambda^{2}\right)^{-N}\left\|\left(1-c(y)^{2}\left(\Delta_{y}+\partial_{x}^{2}\right)\right)^{N} r_{k}(x, y)\right\|_{L^{2}} \\
& \leq C_{N}\left(1+\lambda^{2}\right)^{-N} e^{-\frac{1}{2} k^{\delta}}
\end{aligned}
$$

which finishes the proof.

\section{References}

[A] S. Agmon, Lectures on Exponential Decay of Solutions of Second Order Elliptic Equations, Princeton University Press, Princeton, N.J., 1982.

[D] E.B. Davies, Spectral properties of compact manifolds and changes of metric, Amer. J. Math. 112 (1990), 15-39.

[G] D. Grieser, $L^{p}$ bounds for eigenfunctions and spectral projections of the Laplacian near concave boundaries, Thesis, UCLA (1992).

[K] L. Kapitanski, Some generalizations of the Strichartz-Brenner inequality, Leningrad Math. J. 1 (1990), 693-726.

[MSS] G. Mockenhaupt, A. Seeger and C. D. Sogge, Local smoothing of Fourier integrals and Carleson-Sjölin estimates, J. Amer. Math. Soc. 6 (1993), 65-130.

[RS] M. Reed and B. Simon, Methods of Modern Mathematical Physics IV: Analysis of Operators, Academic Press, New York, 1978.

[S] C. D. Sogge, Concerning the $L^{p}$ norm of spectral clusters for second order elliptic operators on compact manifolds, J. Funct. Analysis 77 (1988), 123-134.

[St1] R. Strichartz, A priori estimates for the wave equation and some applications, J. Funct. Analysis 5 (1970), 218-235.

[St2] - Restrictions of Fourier transforms to quadratic surfaces and decay of solutions to wave equations, Duke Math. J. 44 (1977), 705-714.

U Niveriti of Washington, Seatthe

E-mail address: hart@math.washington.edu

University OF CALIFORNiA, Los ANGELES

E-mail address: sogge@math.ucla.edu 